Meta

Journal des traducteurs

Translators' Journal

\title{
Dictionnaire multilingue de médecine - vers une dictionnairique d'apprentissage
}

\section{Madalena Contente et João Magalhães}

Volume 42, numéro 1, mars 1997

Lexicologie et terminologie

URI : https://id.erudit.org/iderudit/002363ar

DOI : https://doi.org/10.7202/002363ar

Aller au sommaire du numéro

Éditeur(s)

Les Presses de l'Université de Montréal

ISSN

0026-0452 (imprimé)

1492-1421 (numérique)

Découvrir la revue

Citer cet article

Contente, M. \& Magalhães, J. (1997). Dictionnaire multilingue de médecine - vers une dictionnairique d'apprentissage. Meta, 42(1), 114-120. https://doi.org/10.7202/002363ar
Résumé de l'article

Le dictionnaire informatisé de médecine en cours d'élaboration a comme destinataires principaux les étudiants de médecine. Il s'agit d'un instrument d'acquisition et de décodage du sens. On détermine les besoins linguistiques à partir d'une analyse sémantique du discours médical, en cherchant les façons d'exprimer les différentes notions utilisées dans ce discours. Ce dictionnaire multilingue (portugais I anglais Ifrançais) rend compte des différents équivalents et conduit à une méthodologie des besoins des utilisateurs, i.e. $\mathrm{T}$ établissement de priorités lexicales, à travers la multiplication d'échanges d'informations internationales en sciences médicales. 


\title{
DICTIONNAIRE MULTILINGUE DE MÉDECINE — VERS UNE DICTIONNAIRIQUE D'APPRENTISSAGE*
}

\author{
Madalena Contente et Jõ̃o MaGalhães** \\ Universidade Nova de Lisboa, Portugal
}

\begin{abstract}
Résumé
Le dictionnaire informatisé de médecine en cours d'élaboration a comme destinataires principaux les étudiants de médecine. Il s'agit d'un instrument d'acquisition et de décodage du sens. On détermine les besoins linguistiques à partir d'une analyse sémantique du discours médical, en cherchant les façons d' exprimer les différentes notions utilisées dans ce discours.

Ce dictionnaire multilingue (portugais / anglais / français) rend compte des différents équivalents et conduit à une méthodologie des besoins des utilisateurs, i.e. l'établissement de priorités lexicales, à travers la multiplication d'échanges d'informations internationales en sciences médicales.

Abstract

Given their intrinsic characteristics, dictionaries are didactic tools since they facilitate acquisition and decoding of meaning. This multilingual Portuguese/English/French medical dictionary, intended primarily for medical students, seeks to provide various equivalents using a methodology based on users' needs by establishing lexical priorities through increased international exchange of information pertaining to the medical sciences. Needs are identified through semantic analysis of medical discourse to discover how various concepts are expressed.
\end{abstract}

\section{INTRODUCTION}

La médecine, telle qu'elle se présente actuellement, a pris naissance au siècle dernier quand les médecins scientifiques commencèrent à explorer systématiquement la structure et les fonctions de l'organisme humain malade et de l'organisme en bonne santé. La rupture avec la tradition antérieure provoqua un malaise considérable qui souleva même des discussions philosophiques dans les revues médicales. Toutefois, jusqu'à la fin du siècle, la profession médicale admit en général que la médecine était une branche des sciences naturelles et que les processus des maladies devaient être expliqués en termes anatomiques et physiologiques (Wulff et al. 1993: 27).

On constate, en se basant sur une réflexion scientifique, que le paradigme d'une science qui touche le sens des concepts fondamentaux (comme la maladie en médecine), les limites du champ de recherche (la frontière entre les problèmes qui sont considérés comme médicaux et ceux qui se rapportent à d'autres disciplines), comprend les théories de base, les méthodes de recherche reconnues et les valeurs auxquelles les chercheurs adhèrent. Les composantes d'un paradigme constituent la connaissance tacite de la communauté scientifique.

Kuhn (1970) a introduit le concept de paradigme d'une science. Pour lui, le paradigme a un sens plus restreint: il s'applique aux résultats des recherches scientifiques qui servent de référence pour des recherches ultérieures. Il définit paradigme qui «d'un côté représente la constellation entière d'opinions, de techniques, etc., qui sont partagées par les membres d'une communauté donnée. D'un autre côté, il constitue un des éléments de 
cette constellation que sont les solutions d'énigmes utilisées comme modèles spécifiques qui serviront de bases pour en résoudre d'autres [énigmes]» (Wulff et al. 1993: 22).

Pour lui, les scientifiques n'apprennent jamais à déchiffrer dans l'abstrait les concepts, les lois et les théories; mais apprennent progressivement à utiliser les outils intellectuels en lisant des ouvrages scientifiques. Les étudiants en médecine et les jeunes médecins apprennent graduellement à penser comme leurs enseignants et, finalement, lorsqu'ils partagent la connaissance de la profession, ils se sentent intégrés au milieu académique et acceptés comme des collègues.

La science moderne se construit, en grande partie, quand elle se rend paradigmatique; ainsi, elle se définit comme le sujet d'une "science normale» de la communauté scientifique.

La médecine comprend une variété de disciplines et on ne peut pas dire que la pensée médicale soit établie sur un seul paradigme. Popper (1985) soutient également que la science est un processus évolutif et que «le scientifique ne fait pas que produire une nouvelle connaissance à l'intérieur du paradigme établi, il est aussi coresponsable d'un ajustement progressif de ce paradigme» (Wulff et al. 1993: 31).

Bref, on dira que la pensée médicale s'établit sur une complexité de paradigmes, relativement aux différentes disciplines.

\section{DICTIONNAIRE MULTILINGUE DE MÉDECINE}

La conception du modèle de Dictionnaire multilingue informatisé de médecine en cours d'élaboration a comme destinataires / utilisateurs prioritaires les étudiants en médecine.

Le dictionnaire, par ses caractéristiques, est un outil didactique car il s'agit d'un instrument d'acquisition d'encodage et de décodage de la signification. Dans une approche dictionnairique, on dira que le dictionnaire spécialisé informatisé a une structure de communication particulière: il s'agit d'un énoncé spécifique, organisé à partir d'un système de règles formelles, de façon à fournir la documentation scientifique qui, à un niveau précis de spécialisation, essaie de fournir les informations les plus complètes possible.

L'énoncé lexicographique de nature scientifique se revitalise en fonction d'une situation pédagogique, c'est-à-dire qu'il devient un discours pédagogique/didactique (Contente 1992: 280).

Selon Lerat (1995: 174-175), «un dictionnaire spécialisé est proprement terminologique s'il se caractérise par les propriétés suivantes: multilingue, limité à une thématique fortement spécialisée, avec indexation sur les notions (numérotées) et sur les unités terminologiques correspondantes (dans chaque langue). Le multilinguisme révèle les notions réellement partagées et celles qui sont liées à une culture dans une langue, il fait donc voir les limites des équivalences (souvent conditionnelles, du fait de l'universalité de la polysémie et de la synonymie)».

\subsection{DESTINATAIRES/UTILISATEURS}

La constatation du besoin de ressources terminologiques conçues d'un point de vue didactique, de plus en plus éprouvé par les professeurs de médecine, est à l'origine du présent projet dè Dictionnaire multilingue de médecine.

Les étudiants en médecine sont généralement de jeunes adultes, à qui est présentée pendant les cours une explication rationnelle en langue maternelle; cependant, lorsque I'utilisation d'une deuxième langue s'impose, elle doit être complétée avec; une méthode uniquement inductive et unilingue au niveau des équivalents qu'ils trouvent dans ce domaine. Le discours médical, dans les ouvrages de référence, est caractérisé par l'ensemble de cohérence de dénominations de connaissances spécialisées; dans ce cas, la terminologie 
de la médecine, qui se définit en relation au domaine de spécialité ou champ notionnel, est faite vers l'unité terminologique ou terme.

Il faut remarquer que les étudiants en médecine travaillent à partir d'ouvrages en langue maternelle, mais aussi à travers des ouvrages en anglais et en français. On signale la perspective de Lerat (1995: 41-42) «...lire un texte spécialisé en langue maternelle suppose un va-et-vient entre un système de valeurs saussuriennes et un système de valeurs sémiotiques non linguistiques. Avec un texte spécialisé en langue étrangère, [...] elle risque d'être ralentie».

Parfois, les étudiants ont des difficultés quand le matériel didactique des matières à apprendre se trouve en langue étrangère seulement, car ils ne connaissent pas les équivalents exacts dans les différentes langues.

\subsection{LE CORPUS MULTILINGUE}

Suivant cette perspective, notre méthodologie va vers la constitution d'un corpus multilingue, qui rassemble des ouvrages spécialisés en portugais ${ }^{1}$, en anglais ${ }^{2}$ et en français ${ }^{3}$, de bonne réputation scientifique et qui sont de parution assez récente pour que l'information qu'ils contiennent soit actualisée. On travaille avec des manuels d'enseignement universitaire, des revues spécialisées, des comptes rendus de colloques. Ce corpus de base est un corpus multilingue de spécialité informatisé qui nous permet de faire l'extraction de termes, de contextes, de collocations, de phraséologies, et de comparer des définitions.

On possède également un corpus de référence, des dictionnaires spécialisés dans ces mêmes langues.

Il faut aussi remarquer qu'on travaille directement avec des spécialistes du domaine (professeurs de médecine) qui valident les termes et les définitions, soit dans la langue source, soit dans la langue cible.

On partage le point de vue d'Amal Jammal (1988: 541), quand elle dit que «Dans le cas d'un vocabulaire bilingue, où les termes sont définis dans une langue, avec l'identification de leurs équivalents dans l'autre langue, les auteurs devront rechercher des ouvrages spécialisés, écrits dans la langue seconde et contenant des contextes définitoires, et procéder ensuite à la superposition des descripteurs pour établir les équivalences. Il leur faudra auparavant s'assurer de la fiabilité de ces ouvrages et s'informer de l'accueil qui leur a été réservé par les spécialistes du domaine.»

\subsection{LES ÉQUIVALENTS}

La détermination des besoins linguistiques s'effectue à partir d'une analyse sémantique du discours médical; en cherchant les différentes notions, on obtient les termes correspondants utilisés dans ce discours.

Les utilisateurs de ce dictionnaire peuvent recourir à une recherche en langue maternelle afin de maîtriser cette langue de spécialité en ce qui concerne la terminologie du domaine, la variante, la synonymie, la phraséologie, mais ils peuvent aussi trouver les équivalents en langue étrangère (anglais, français).

Dans une perspective linguistique, on soulignera l'importance de l'aspect dynamique de la recherche des équivalents parmi les éléments linguistiques d'une ou de plusieurs langues. La fonction première d'un texte scientifique est la fonction cognitive. Parmi les éléments du contenu (éléments sémantiques), les concepts véhiculés sont très importants.

D'après Kocourek (1991: 212) «si l'étude terminologique comparative (contrastive) explore les rapports entre la terminologie d'un domaine dans deux langues, elle doit avant tout chercher l'équivalence cible du terme source: c'est ce que nous appelons recherche d'équivalents ou recherche des équivalents». 
Les internationalismes de langue spécialisée, surtout ceux d'origine gréco-latine (fréquents dans la terminologie médicale), peuvent être une aide à l'étudiant faisant l'étude systématique des formants internationaux, ils l'aident à comprendre les termes jusque-là inconnus tout en représentant un support important à la mémorisation des terminologies. «En outre, la comparaison critique entre termes de différentes langues lui montre que l'identité de la forme ne permet pas de conclure sans réserve à l'identité du contenu» (Arntz 1988: 537).

Selon Jammal (1988: 537) quand «Il reste à choisir les outils et les matériaux [...] il faut, en premier lieu, une méthodologie rigoureuse [...] [à partir] de la conception de l'arbre terminologique $[. .$.$] que le vocabulaire doit desservir».$

La plupart des dictionnaires spécialisés sont des dictionnaires bilingues ou multilingues. Selon Heid (1988: 226), trois types d'informations indispensables à la description d'équivalences de termes doivent obligatoirement figurer dans ces dictionnaires :

1. informations concernant le domaine d'application d'un terme (ce qui constitue une partie de l'information pragmatique);

2. informations concernant les marques d'usage particulières, qui décrivent les règles régissant l'application d'un terme dans un contexte donné (autre partie de l'information pragmatique);

3. informations concernant les combinaisons possibles entre différents termes (syntagmes nominaux, collocations, etc.), (partie, souvent négligée, de l'information syntaxique et sémantique).

La problématique des équivalents dans les dictionnaires multilingues, comme c'est le cas en médecine, requiert la recherche d'une équivalence totale au niveau sémantique.

Svensén (1993: 143-147) se rapporte aux différentes formes d'équivalence présentées par Kromann (1988: 188), l'équivalence totale, l'équivalence partielle et la non-équivalence, mais il en expose aussi d'autres dont quelques-unes sont indiquées ici :

- l'équivalence métalinguistique;

- l'équivalence à partir d'une information encyclopédique;

- l'équivalence dénotant une super-ordonnance ou des concepts subordonnés;

- l'équivalence sans correspondance;

- l'équivalence alternative (phrases).

Voici maintenant des exemples d'équivalences relevés dans l'étude en cours :

* Des équivalents présentant une fidélité sémantique (Kocourek 1991 : 209) qui se caractérisent par une transparence au niveau graphique, phonologique, syntaxique et sémantique; on dira qu'il s'agit de termes qui ne présentent aucun problème au niveau de la compréhension pour les étudiants.

$\begin{array}{lll}\text { Portugais } & \text { Anglais } & \text { Français } \\ \text { Abulia } & \text { Abulia } & \text { Abulie } \\ \text { Candidiase } & \text { Candidiasis } & \text { Candidose } \\ \text { Glutamina } & \text { Glutamine } & \text { Glutamine } \\ \text { Hamartoma } & \text { Hamartoma } & \text { Hamartome }\end{array}$

* Des équivalents qu'on dénomme sémantiquement complets, mais qui présentent une forme variée dans les différentes langues. Ce sont des termes comme ceux-là qui présentent des problèmes, surtout pour les étudiants qui ne maîtrisent pas totalement une des langues, comme langue de spécialité. 


\section{Portugais}

Cadeias pesadas

(doença das)

Enterobiase

Hematoma

retroplacentário
Anglais

Franklin's disease

Enterobiasis

Abruptio placentae
Français

Chaînes lourdes

(maladie des)

Oxyurose

Hématome

rétroplacentaire

* Enfin, les étudiants peuvent trouver aussi dans les différents ouvrages plusieurs équivalents pour le même terme. C'est la problématique des variantes synonymiques et des synonymes partiels, qui peuvent poser des difficultés de compréhension quand ils rencontrent plusieurs termes pour le même concept. À notre avis, toutes ces variantes synonymiques doivent être toujours enregistrées dans le dictionnaire.

$\begin{array}{lll}\text { Portugais } & \text { Anglais } & \text { Français } \\ \text { Gonorreia } & \text { Gonorrhea } & \text { Gonorrhée; } \\ \text { Blenorragia } & & \text { Blennorragie; } \\ \text { Leucorreia } & & \text { Gonococcie; } \\ \text { Bartonelose } & \text { Bartonellosis } & \text { Bartonellose; } \\ & \text { Carrion's disease } & \text { Carrion (maladie de) }\end{array}$

«En présentant diverses dénominations d'un même concept, dans plusieurs langues, et en explicitant les signifiants, on apporte sur le concept un éclairage neuf. Il est évident que cette activité de type linguistique ne peut se substituer à l'acquisition des concepts dans le cadre de leur discipline» (Thoiron 1994: 771).

L'objectif prioritaire de ce travail doit prendre en compte ce que Rousseau (1995:3) dénomme la qualité du point de vue du locuteur, qui doit comprendre:

- l'accessibilité : l'usager doit pouvoir trouver rapidement la terminologie appropriée à ses besoins et adaptée au niveau et à la situation de communication;

- l'actualité: quel que soit le domaine ou le champ d'expérience, le locuteur doit pouvoir utiliser une terminologie à jour ;

- la fiabilité : l'usager doit utiliser une terminologie reconnue par ses pairs et par le milieu professionnel auquel il s'adresse, s'il veut communiquer efficacement.

Toutes ces questions d'équivalence s'avèrent d'une importance primordiale dans la construction d'un dictionnaire dans une perspective dictionnairique didactique, qui concerne les besoins langagiers du destinataire/utilisateur/étudiant en médecine.

\section{VERS UNE APPROCHE DICTIONNAIRIQUE D'APPRENTISSAGE}

La compréhension, conçue par la psychologie cognitive classique en tant que théorie des représentations mentales, se présente comme la construction des représentations sémantiques formalisées par des graphes conceptuels.

En affirmant que les performances dépendent des caractéristiques des représentations construites au cours de la lecture, on envisagera la mémoire, non comme un système passif d'enregistrement et de stockage, mais comme un système dynamique de construction et de transformation de structures cognitives qui devront pouvoir être réactualisées.

On peut distinguer différentes approches (cf. Kocourek 1988): une approche contrastive, qu'on considère comme didactique, est, dans ce cas-là, multilingue, synchronique, basée sur la langue maternelle comme langue de départ (thématique) vers une langue seconde comme langue cible (rhématique).

Une approche interférentielle, au sens linguistique; qui regroupe les problèmes linguistiques du bilinguisme ou du multilinguisme, des langues en contact et de l'emprunt (cf. Weinreich 1969, 1970; et Deroy 1956 cité par Kocourek 1988: 547). 
Une approche lexicographique, car il s'agit d'une comparaison multilingue, synchronique, lexico-sémantique, dont le but est de faciliter la compréhension et l'équivalence, qui sert de source, d'une part, à une théorie de lexicographie multilingue et, d'autre part, à une comparaison lexico-sémantique structurelle des trois langues où l'on cherche surtout l'équivalence paradigmatique des unités terminologiques et de leurs acceptions variées.

L'intérêt de l'approche multilingue en terminologie est très important, comme le soutient Thoiron (1994: 769): «...selon la langue, on éclaire, par le biais de la nomination, tel(s) ou tel(s) élément(s) constitutif(s) du concept. En proposant, pour un concept donné, des équivalents en plusieurs langues, on enrichit la description des éléments du concept puisqu'on allonge la liste des éléments dénommés».

Selon une étude présentée par Garcia (1994: 151-160)4, le progrès scientifique déterminé par une communication permanente parmi les chercheurs aboutit à la fixation d'une ressemblance au niveau morpho-sémantique et syntaxique dans les différents vocabulaires des systèmes linguistiques internationaux. Cette identité est fondamentale, surtout s'il s'agit de terminologies à racines grecques et latines, rendant le langage assez universel et facilement reproductible d'une langue à l'autre.

Les langues spécialisées qui ont un processus d'emprunt mutuel acquièrent des structures propres soit au niveau lexical, soit au niveau de la morphogenèse, soit au niveau de la structure syntaxique; cela aboutit à ce qu'on peut dénommer interlangue - moyen de communication parmi les différentes langues du même domaine de spécialité

L'élaboration de ce dictionnaire spécialisé dans une perspective d'approche dictionnairique d'apprentissage prend surtout en compte les besoins spécifiques de l'apprenant/ étudiant en médecine. Il pourra mener les recherches qu'il jugera nécessaires, c'est-à-dire les termes qu'il ne maîtrise pas totalement, lesquels se trouvent dans le dictionnaire avec l'information la plus complète et la plus précise possible; le dictionnaire aura une composante textuelle qui pourra contribuer au processus d'apprentissage terminologique. Cette étude de fabrication du dictionnaire envisage la nature du discours scientifique médical utilisé par les spécialistes qui ont une performance communicative spécialisée, prenant en compte les tendances actuelles des principes socioterminologiques.

Pour conclure, le Dictionnaire multilingue (portugais, anglais, français) se base sur une approche méthodologique qui prend comme point de départ les besoins langagiers des utilisateurs. Il établit des priorités lexicales à travers la multiplication des échanges d'informations internationales au niveau de la science médicale, communication internationale scientifique qui a comme base de compétence la communication socioprofessionnelle.

\section{Notes}

* Este trabalho foi apoiado pelo Instituto Camões e pela JNICT através do Programa Lusitânia.

* Cet article est issu d'une communication présentée par l'auteur aux IV Journées scientifiques du réseau «Lexicologie, terminologie, traduction» de l'AUPELF-UREF (Lyon, France, 28, 29, 30 septembre 1995).

1. ANTUNES, Abílio B. S. et al. (1984): Manual de Urgências, Lisboa, Ed. Sandoz-Warner.

2. HARRISON'S (1991): Principles of International Medicine, Wilson, Braunwald, Isselbacher, Petersdorf, Martin, Fauci, Root (Eds), New York. International Edition, vol, 1 et 2, $12^{\mathrm{e}}$ édition, McGraw-Hill inc.

3. MATHÉ, G. et G. RICHET (1981) : Sémiologie médicale, Paris, Flammarion Médecine-Sciences, $4^{\mathrm{e}}$ édition, tomes I, II, III.

4. Cf. le point 2.4.5. Intersistema-Interlangue, pp. 151-160. 


\section{RÉFÉRENCES}

ARNTZ, R. (1988) : «Le rôle des internationalismes dans la formation des traducteurs», Meta, 33 (4), Montréal, Presses de l'Université de Montréal, pp. 497-504.

BO SVENSÉN (1993): Practical Lexicography, translated by John Sykes and Kerstin Schofield, Oxford (NY), Oxford University Press.

CONTENTE, M. (1992): Do Autodicionário ao Dicionário de Turma Informatizado - Domínio de Aplicąāo Biologia: Reprodução e Hereditariedade, Tese de Mestrado em Linguística, Área de especialização Lexicologia e Lexicografia, Faculdade de Ciências Sociais e Humanas, Universidade Nova de Lisboa, Lisboa.

CONTENTE, M. et J. MAGALHÃES (1994) : «A Terminologia da Medicina - A Problemática dos Equivalentes», V Congresso Luso-Hispano de Lenguas Aplicadas a las Ciencias, Universidade de Valencia, 29, 30 Set. e 1 Out., Actes à paraître.

GARCIA, L. (1994): Terminologia da Senologia - Projecto de um dicionário interactivo multilingue - Para uma aprendizagem da língua de especialidade, Tese de Mestrado em Linguística, Área de especialização Lexicologia e Lexicografia, Faculdade de Ciências Sociais e Humanas, Universidade Nova de Lisboa, Lisboa.

GHAZI, J. (1983) : Contenu lexical et apprentissage du vocabulaire domaine du français médical, thèse de Doctorat, Université Sorbonne Nouvelle, Paris.

HEID, U. (1988): «Attentes des terminologies à l'égard des programmes d'élaboration des dictionnaires», Terminologie diachroniques, Bruxelles, CILF, pp. 221-242.

JAMMAL, A. (1988) : «Les vocabulaires des spécialités médicales : pourquoi et comment les fabrique-t-on ?», Meta, 33 (4), Montréal, Presses de l'Université de Montréal, pp. 535-541.

KOCOUREK, R. (1988): «Les comparaisons linguistiques et la comparaison bilingue intégrale», Meta, 33 (4), Montréal, Presses de 1'Université de Montréal, pp. 542-549.

KOCOUREK, R. (1991): La langue française de la technique et de la science, $2^{\mathfrak{e}}$ édition, Wiesbaden, Oscar Brandstetter, GMBH \& CO.KG.

KROMANN, H.-P. (1983) : «Pradignatische und syntagnatische Relationen in Zweisprachigen Wöterbuch», Die Lexikographie von heute und das Wörterbuch non morgen, J. Schildt et D. Viehragen (Eds), Linguistische Studien, Akademic der Wisseuschaften der DDR, Berlin.

KUHN, T. (1970) : La structure des révolutions scientifiques, Paris, Flammarion.

LERAT, P. (1995) : Les langues spécialisées, Paris, PUF, coll. «Linguistique Nouvelle».

POPPER, K. (1985): Conjonctures et réfutations: la croissance du savoir scientifique, Paris, Payot.

ROUSSEAU, L.-J. (1995): Principes méthodologiques du travail terminologique, document présenté au Réseau Panlatin de terminologie, avril 1995, Québec, 7 p.

SVENSÉN, Bo (1993) : Practical Lexicography, Oxford, OUP.

THOIRON, Ph. (1994) : «La terminologie multilingue : une aide à la maîtrise des concepts», D. Candel, M. Cormier et J. Humbley (dir.), Meta, 39 (4), numéro spécial «Termes et textes», Hommage à Bernard Quemada, Montréal, Presses de l'Université de Montréal, pp. 765-773.

WULFF, PEDERSON et ROSENBERG (1993): Invitation à la philosophie de la médecine, traduction et préface de A. C. Masquelet, Montpellier, Sauramps médical. 